\title{
Underwater Wireless Communication System
}

\author{
Prof. Ranjitha Rajan \\ Electronics and Communication Department \\ Amal Jyothi College of Engineering \\ Kottayam, India \\ Ms. Amala Susan Roy \\ Electronics and Communication Department \\ Amal Jyothi College of Engineering \\ Kottayam, India
}

\author{
Ms. Elizabeth P.T \\ Electronics and Communication Department \\ Amal Jyothi College of Engineering \\ Kottayam, India \\ Ms. Aiswarya K.S \\ Electronics and Communication Department \\ Amal Jyothi College of Engineering \\ Kottayam, India
}

\author{
Ms. Christymol Bousally \\ Electronics and Communication Department \\ Amal Jyothi College of Engineering \\ Kottayam, India
}

\begin{abstract}
Water data communication is a potential technology to realize underwater communication. The experiment of underwater data communication in the laboratory is different with that in the real water environment because the physical scale is limited. In this paper, several kinds of agents are evaluated to change the coefficients of experimental water precisely. Then, seemed as criterion for the reliability of water recreation, the frequency domain characteristic of data communication through water channel in experimental water is measured and compared. The results show that the type and particle size of the agents will significantly affect its water properties, and the frequency domain component of the water communication signal will be affected by the agent's concentration. By having a separate transmitter and receiver module in the water between the modules we can transmit the sea researcher's biomedical conditions and interactions to the monitoring end available on the ship. The atmospheric conditions under the sea will be changing tremendously. It affects the sea navigators in too much trouble. It is difficult to monitor the health conditions of sea navigator after diving. Even if he dives below 20 meters his heart beat becomes uncontrollably changing. So even if his blood pressure lows dangerously it may leads to death. And there is no idea to save those navigators in those conditions. So here we propose a system to monitor heath conditions of a sea navigator while checking the heart beat reading while navigation. Using a communication system we can monitor a diving navigators heart beat even in ground. If we found his BP is lower in we can say him to enter the ground. So we can save the life of too many navigators.
\end{abstract}

Keywords- Underwater communication, wireless optical communication, scuba diving, heart beat sensor.

\section{INTRODUCTION}

Underwater wireless information transmission is of countless importance to the military, industry and the scientific community. So as to facilitate all these activities, there is a rise in the number of unmanned vehicles or devices deployed underwater, which require high bandwidth and high capacity for information transfer underwater. Within the previous couple of years, the interest towards optical wireless communication has increased for terrestrial, space and underwater links as it is capable of providing high data rates with low power and mass requirement. Many of researchers have administered work for terrestrial and space links, however underwater optical wireless links are relatively less explored because it is tougher than atmospheric links. The main difficulty for reliable underwater communication is due to wide selection of physical processes in various sorts of underwater environments starting from shallow coastal water to deep sea or oceans. Optical communication may be a budding technology to understand underwater wireless communication.

The experiment of underwater optical communication within the laboratory is different within the real water environment because the physical scale is restricted. Although since recent several decades, artificial scattering agents are conditioned to recreate underwater optical communication channels under different water quality conditions, but the similarity between experimental water and natural water isn't reliable, like the similarity in frequency domain characteristics. Facing the problem of alignment caused by uncertainty of the position of transmitter and receiver, poor mechanical stability along with the complexity of water environment, the transmission characteristics of underwater optical communication signals under alignment conditions are difficult to get within the natural seawater environment.

An acoustic communication has been developed for the underwater wireless sensor network because of its relatively low attenuation, but the bandwidth of the underwater acoustic channel is restricted, making it less favourable for data-intensive applications. Besides, the slow propagation of underwater sound also results in a considerable delay within the underwater communication link. Underwater sensors cannot share data with those ashore, as both use different wireless signals that only labour in their respective mediums. Radio signals that 
travel through air die very quickly in water. Acoustic signals, or sonar, sent by underwater devices generally reflect off the surface without ever breaking through. This causes inefficiencies and other issues for a diversity of applications, such as ocean exploration and submarine-toplane communication. So as to beat the disadvantages in the underwater acoustic communication, a replacement approach which is in the most rising area of research is that the underwater wire-less optical communication. Initial research has begun to determine an immediate communication link between the satellite or aircraft and the underwater vehicle. Recently, underwater wireless optical communication using cheap light-emitting diodes and laser diodes also has been researched. Comparing the light sources utilized in these underwater wireless optical communication systems, it is apparent that the LD outperforms light-emitting diodes in terms of knowledge rate. Although it is difficult to transmit long distance, it is possible to unravel the matter of interference between symbols associated with multi-path propagation and therefore the security problem associated with eavesdropping employing a point-to-point method that exploits the laser's characteristics of strong directivity at a brief distance. The blue-green laser has the minimum energy fading within the sea, whose fading rate is approximately $0.155-0.5 \mathrm{~dB} / \mathrm{m}$. Hence, exploiting the low absorption window of seawater within the blue-green portion of the electromagnetic spectrum, underwater wireless laser communication is estimated to play an important role by offering secure, efficient, and high data rate communication within short distances.

Normally sea navigators or fisherman require an additional support from the land when something uncharacteristic things happen within the sea, we have to pass the message to them. With development of the technologies our people can predict what happen in the sea, but to transfer these messages to sea navigators or fisherman is difficult. So as to avoid these issues we introduce an enhanced system named under water data communication. This paper discusses about the underwater data communication system used for transferring messages to sea navigators or fishermen and also as we can monitor their health conditions. We use water data communication module to get the text and sensor readings to the ground from navigator and this will help us to avoid the undesirable death of navigators undersea. Since the conditions of underground water even temperature, pressure may vary in any condition; it also contrasts the body temperature, blood pressure of navigator. So if it lowers or higher during a wide selection, it cause even the death of the navigator. It also provides the needed medical aid to the concerned person. It is applicable for staffs of forces, marine drivers and other people who participate in water entertainments. This is often what we have accomplished with wireless underwater communications. As against hardwired communications which confines you to the length of a cable, wireless communications provides you the facility to communicate with your buddy, or top side, with ease and freedom to easily dive. Wireless underwater communications are a stimulating and easy way to freely stay connected underwater.

\section{II.MOTIVATION AND PROBLEM STATEMENT}

\section{A. Existing System}

Almost no other systems to watch the health conditions of sea navigator while navigating the sea. While there is a wearable device for monitoring his/her pulse for himself/herself. But an individual within the ground cannot find about the health conditions of person underwater. So he does not realize the health conditions of the person underwater.

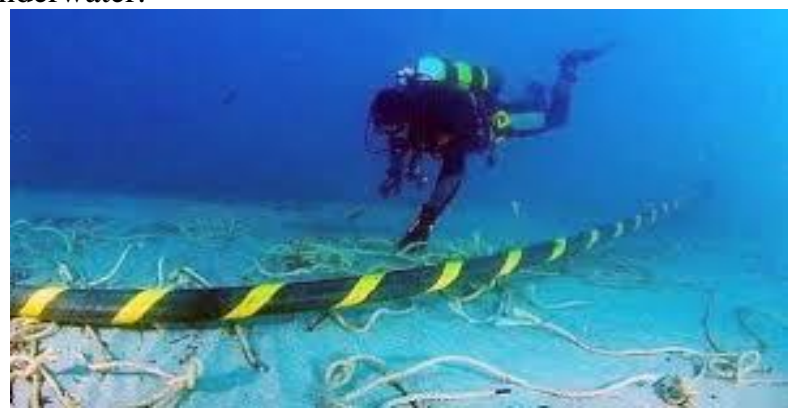

Fig 1: A Scuba Diver

Diving equipment is equipment employed by underwater divers to form diving activities possible, easier, safer and comfortable. This might be equipment chiefly intended for this purpose, or equipment intended for other purposes which is found to be suitable for diving use.

The fundamental item of diving equipment is an underwater breathing device, and surface supplied diving equipment. But there are no other important pieces of kit that make diving safer, more convenient or more efficient. Diving equipment employed by recreational scuba divers is usually personal equipment carried by the diver, but professional divers, particularly when functioning within the surface supplied or saturation mode, uses a good deal of support equipment not carried by the diver.

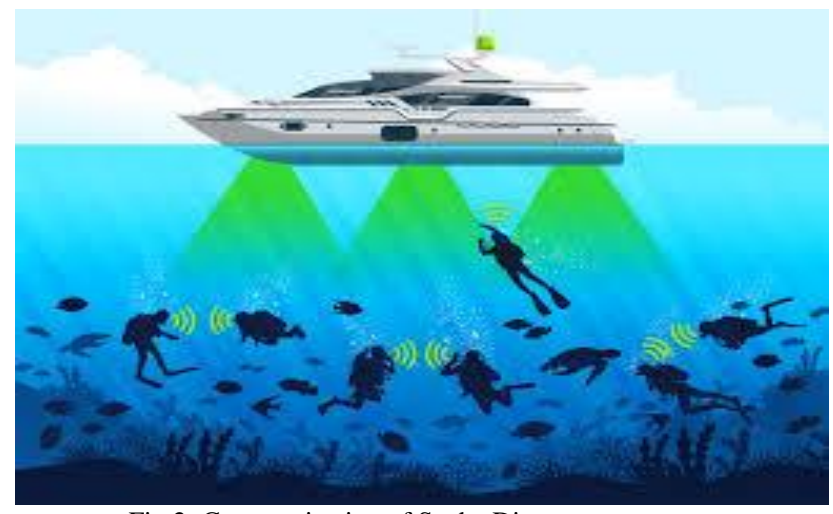

Fig 2: Communication of Scuba Diver

Scuba diving is a mode of underwater diving. Here, the diver uses a self-contained underwater breathing device (scuba), to breathe underwater. Scuba divers carry their own source of breathing gas, usually compressed gas. 
It allows them greater independence and freedom of movement. Although the utilization of compressed air is common, a new mixture called enriched air (Nitrox) has been gaining popularity due to its advantage of reduced nitrogen intake during repetitive dives. Open circuit scuba systems contains one or more diving cylinders comprising of breathing gas at high pressure which is supplied to the diver via a regulator. They may include extra cylinders for range extension, decompression gas or emergency breathing gas. Closed-circuit or semi-closed circuit rebreather scuba systems sanction the recycling of exhaled gases. The volume of gas used is reduced compared to that of open circuit, so a smaller cylinder or cylinders could also be used for equivalent dive duration. Rebreathers lengthen the time spent underwater compared to circuit for corresponding gas consumption; they produce fewer bubbles and fewer noise than circuit scuba. It makes them attractive to hidden military divers to avoid detection, scientific divers to avoid disturbing marine animals, and media divers to avoid bubble interference.

Disadvantages of the prevailing system are the person cannot communicate with the ground and the conditions of his health cannot be monitored in the sea. Even if he dies, the people in the ground will not be able to realize it. He also would not be able to know about anything taking place in the ground.

\section{B. Proposed System}

Today, underwater sensors cannot share data with those ashore, as both use different wireless signals that only work in their corresponding mediums. Radio signals that travel through air die very quickly in water. Acoustic signals, or sonar, sent by underwater devices generally reflect off the surface without ever breaking through. This causes inefficiencies and other problems for a variety of applications, like ocean exploration and submarine-to-plane communication.

To start, there are two selections of underwater communications: Hardwired- uses a cable to transfer the communications; Wireless- uses the water to transfer the communications. Many divers prefer the idea of freely diving where they please with the ability to communicate to their dive partner easily and quickly.

This is what we have accomplished with wireless underwater communications. As opposed to hardwired communications which limits you to the length of a cable, wireless communications provides you the ability to communicate with your companion, or top side, with ease and freedom to simply dive. Wireless underwater communications are an interesting and easy way to freely stay associated underwater.

Advantages of this system are a person who is in the ground can observe the health conditions of navigator, alert messages can pass to navigators from the ground, navigators/fishermen can communicate with ground, and his health conditions can be monitored anywhere, ease of deployment, aptness of data and reliability.

\section{SYSTEM MODEL}

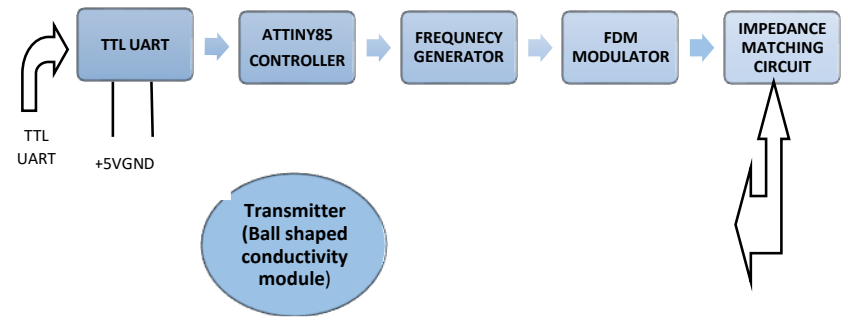

Fig 3: Transmitter Module

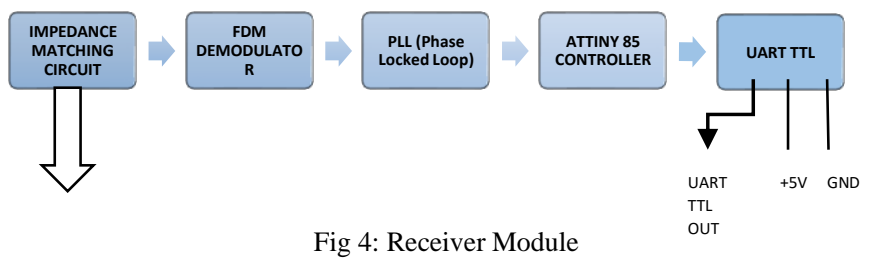

Fig 4: Receiver Module

As shown in the block diagram below, our system comprises of a couple of Microcontrollers, Heart beat sensor, temperature sensor and Data Communication Module. We divided the system into two parts; Transmitter module and receiver module. In the Transmitter end, we have a microcontroller, LCD Display, Heart beat sensor, Temperature sensor and data communication module. Then receiver module consists of Microcontroller, LCD display and Data communication receiver module. Through our system we transfer data (Text/Image) from one end to another end via data communication module. At the beginning, the readings were taken from heart beat sensor and body temperature sensor and displayed on LCD display of transmitter end. Once we dipped the data communication module in the water, the controller in the transmitter side transmits the data to the receiver side through water via data communication transmission module. Here water will act as a medium between transmitter and receiver. Water data communication receiving module will receive the data and provides to the controller in the receiver section. Then the data is displayed in the LCD display of the Receiver end. Through this we can get to know the condition of the navigator. 


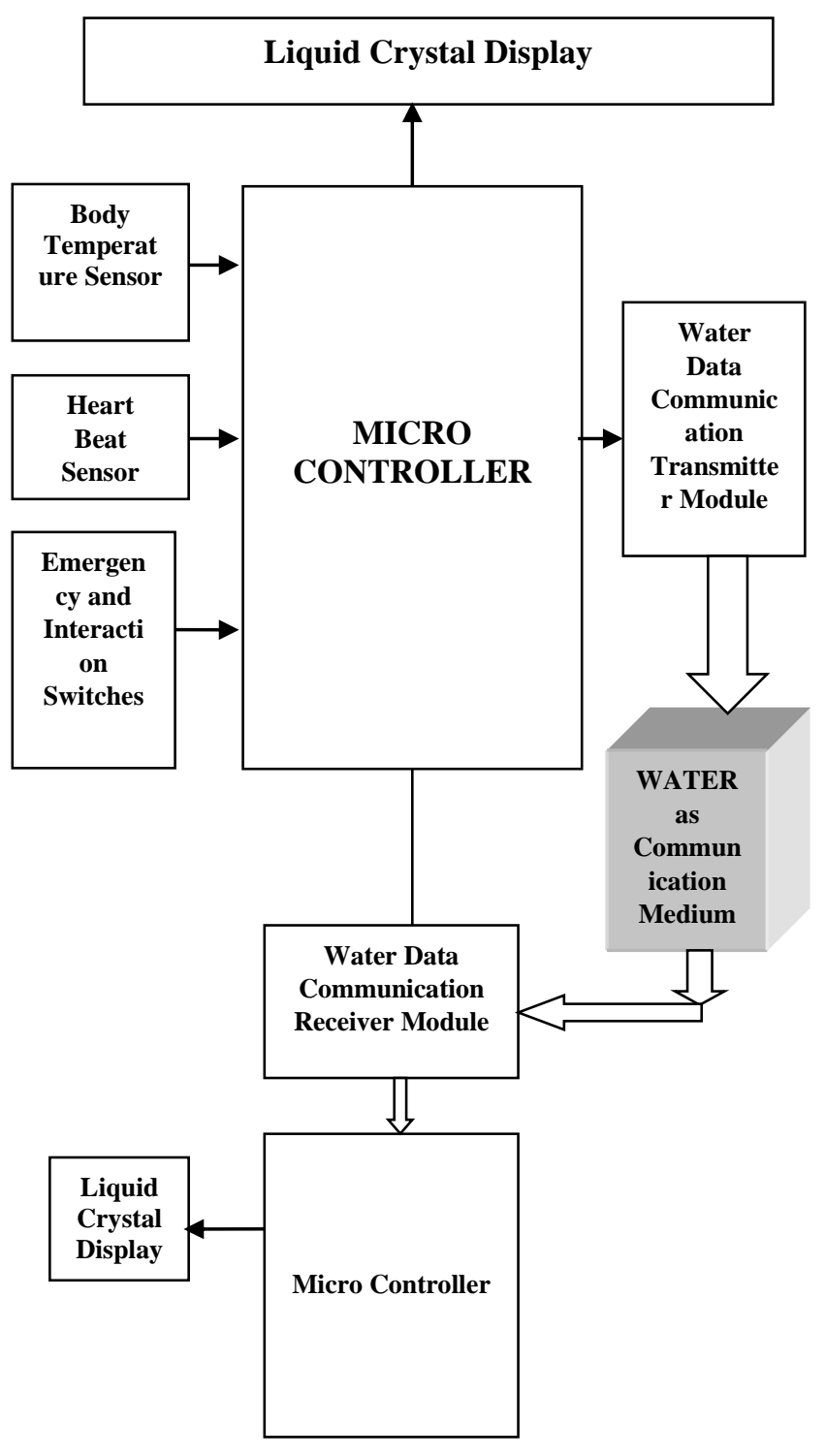

Fig 5: Block Diagram

Next if we want to transfer any data to the navigator or navigator wants to share anything to the ground, we can use this system. We have an application which will help us to transfer the messages. We can type the message in the application and can transfer the message to another end via data communication module. If we want to send some images to the other end, we can choose those images and can send through this application. The Image/Text data will receive by microcontroller and will transfer the data via data communication module through water as medium. Through this communication we can always help the fishermen/ navigators before something happens.

Hardware specifications are Ardunio Uno, Atmega328 Microcontroller, Body Temperature Sensor, Heart Beat Sensor, Water Data Communication Receiver \& Transmitter, Jumper Wire and LCD display.

\section{A. Arduino Controller Board}

Arduino is associate American Standard Code for Information Interchange file hardware and computer code package company, project and user community that styles and manufactures single-board microcontrollers and microcontroller kits for building digital devices. Its product are accredited underneath the wildebeest Lesser General Public License (LGPL) or the wildebeest General Public License (GPL), allowing the manufacture of Arduino boards and software package distribution by anyone. Arduino boards are offered commercially in preassembled type or as homemade (DIY) kits.

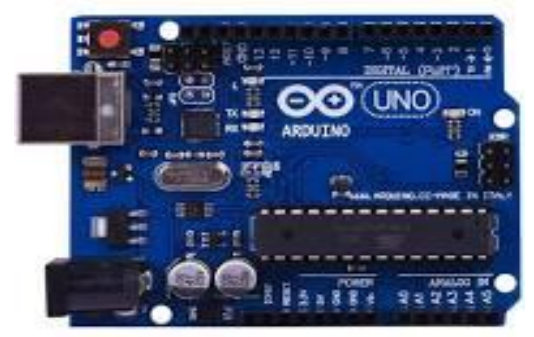

Fig 6: Arduino Controller Board

Arduino board styles use a range of microprocessors and controllers. The board's area unit equipped with sets of digital and analog input/output (I/O) pins which will be interfaced to numerous enlargement boards ('shields') or breadboards (For prototyping) and different circuits. The board features serial communications interfaces, as well as Universal Serial Bus (USB) on some models that also is used for loading programs from personal computers. The microcontrollers may be programmed victimisation $\mathrm{C}$ and $\mathrm{C}++$ programming languages. Additionally, to victimisation ancient compiler tool chains, the Arduino project provides AN integrated development surroundings (IDE) supported the process language project

\section{B. Atmega328 Microcontroller}

Arduino Uno could be a microcontroller board. It's a fourteen digital input/output pins (of that vi are often used as PWM outputs), via analog inputs, a sixteen megacycle ceramic resonator (CSTCE16M0V53-R0), a USB association, an influence jack, an ICSP header and a push button. It contains everything required to support the microcontroller; merely connect it to a pc with a USB cable or power it with an AC to DC adapter or battery to urge started. Within the worst case situation we will replace the chip for a couple of greenbacks and begin once more. "Uno" means that one in Italian and was chosen to mark the discharge of Arduino package (IDE) 1.0. The Uno board and version 1.0 of Arduino package (IDE) were the reference versions of Arduino, currently evolved to newer releases. The Uno board is that the 1st in a very series of USB Arduino boards, and also the reference model for the Arduino platform; for an intensive list of current, past or superannuated boards see the Arduino index of boards. 
The ATmega328 may be a single-chip microcontroller created by Atmel within the mega AVR family (later chip Technology non-inheritable Atmel in 2016). It's a changed Harvard design 8-bit reduced instruction set computing processor core. The Atmel 8-bit AVR RISC-based microcontroller combines thirty two computer memory unit ISP non-volatile storage with readwhile-write capabilities, one computer memory unit EEPROM, 2 KB SRAM, twenty three general purpose I/O lines, thirty two general purpose operating registers, 3 versatile timer/counters with compare modes, internal and external interrupts, serial programmable USART, a byteoriented 2-wire serial interface, SPI interface, 6-channel 10-bit A/D convertor (8-channels in TQFP and QFN/MLF packages), programmable watchdog timer with internal generator, and 5 code selectable power saving modes. The device operates between 1.8-5.5 volts. The device achieves outturn approaching one unit per megahertz.

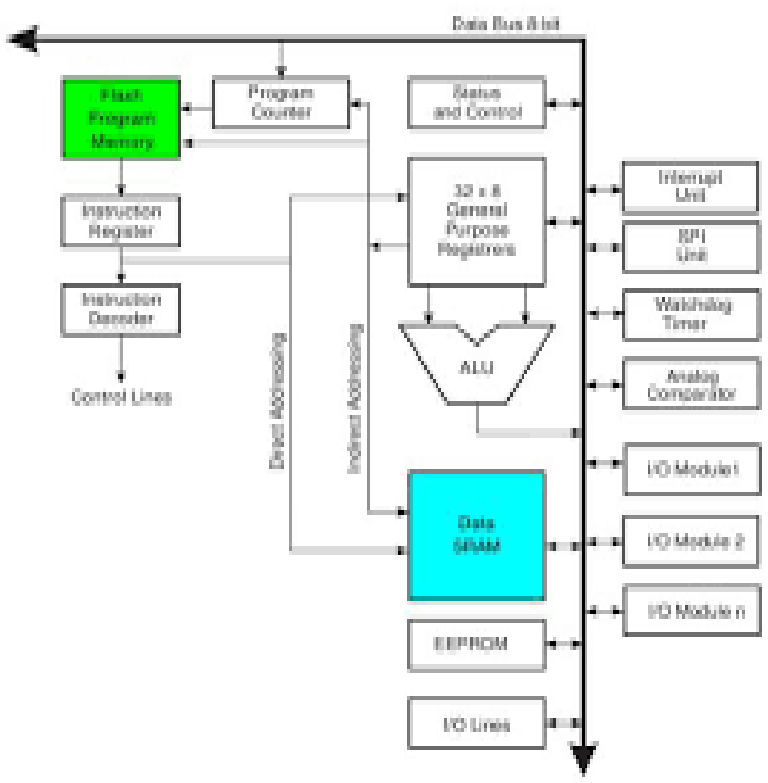

Fig 7: Atmega328 Microcontroller

\section{Heart Beat Sensor}

Pulse detector may be a well-designed plug-and-play heartrate detector for Arduino. It will be used by students, artists, athletes, makers, and game \& mobile developers WHO wish to simply incorporate live heart-rate knowledge into their comes. It conjointly includes Associate in Nursing ASCII text file watching app that graphs your pulse in real time.

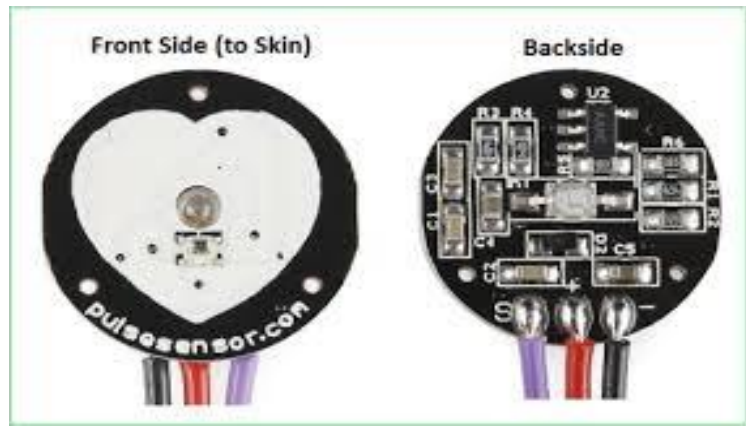

Fig 8: Heart Beat Sensor
The normal resting vital sign for adults over the age of ten years, together with older adults, is between sixty and one hundred beats per minute (bpm). Extremely trained athletes could have a resting vital sign below sixty beats per minute, typically reaching forty beats per minute. The resting vital sign will vary among these traditional vary.

\section{Temperature Sensor}

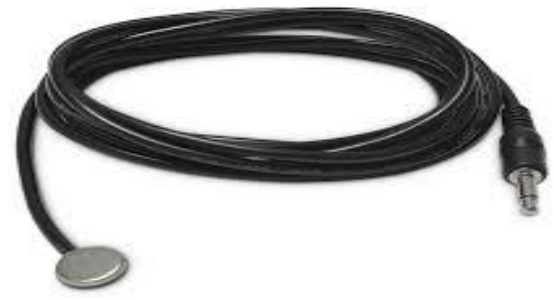

Fig 9: Temperature Sensor

Infrared (IR) temperature sensors alter correct noncontact temperature measuring in medical applications. The foremost common application for this sort of temperature sensing element is mensuration ear temperature, forehead temperature, or skin temperature. Temperature sensing element could be a device won't to live the temperature through associate electrical signal it needs a thermometer or RTD (Resistance Temperature Detectors). If the distinction in voltage is amplified, the analogue signal is generated by the device and it's directly proportional to the temperature.

\section{E. Water Data Communication Module}

Used for digital communication between transmitter and receiver section through the medium of water. The signals square measure regenerate into pulses and undergone the water from one finish to a different.

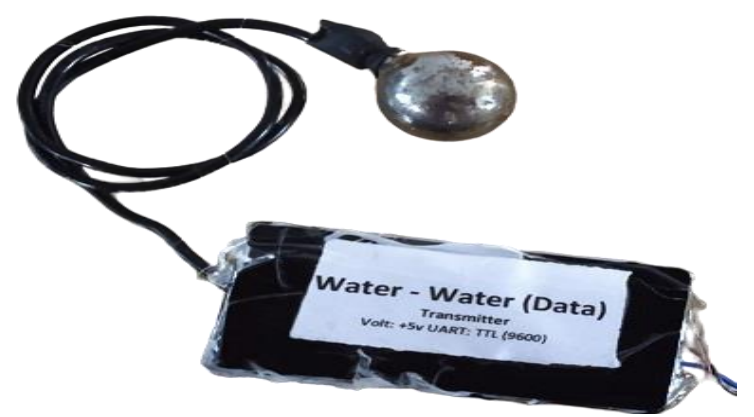

Fig 10: Water Data Communication Module

\section{SOFTWARE SPECIFICATIONS}

\section{A. Arduino IDE}

The Arduino Integrated Development Environment (IDE) is a cross-stage application (for Windows, macOS, Linux) that is written in capacities from $\mathrm{C}$ and $\mathrm{C}++$. It is utilized to compose and transfer projects to Arduino viable sheets, yet additionally, with the assistance of outsider canters, other merchant improvement sheets. 
The source code for the IDE is delivered under the GNU General Public License, form 2. The Arduino IDE underpins the dialects $\mathrm{C}$ and $\mathrm{C}++$ utilizing extraordinary guidelines of code organizing. The Arduino IDE supplies a product library from the Wiring venture, which gives numerous regular information and yield systems. Client composed code just requires two fundamental capacities, for beginning the sketch and the primary program circle, that are incorporated and connected with a program stub principle() into an executable cyclic chief program with the GNU tool chain, likewise included with the IDE dispersion. The Arduino IDE utilizes the program avrdude to change over the executable code into a book document in hexadecimal encoding that is stacked into the Arduino board by a loader program in the board's firmware. Naturally, avrdude is utilized as the transferring instrument to streak the client code onto official Arduino boards. In October 2019 the Arduino association started giving early admittance to another Arduino Pro IDE with debugging and other progressed highlights.

\section{B. Proteus Design Suite}

The Proteus Design Suite is an exclusive programming device suite utilized principally for electronic structure computerization. The product is utilized mostly by electronic plan designers and experts to make schematics and electronic prints for assembling printed circuit sheets. It was created in Yorkshire, England by Labcenter Electronics Ltd and is accessible in English, French, Spanish and Chinese languages. Schematic catch in the Proteus Design Suite is utilized for both the recreation of plans and as the structure period of a PCB format venture. It is along these lines a center part and is incorporated with all item designs.

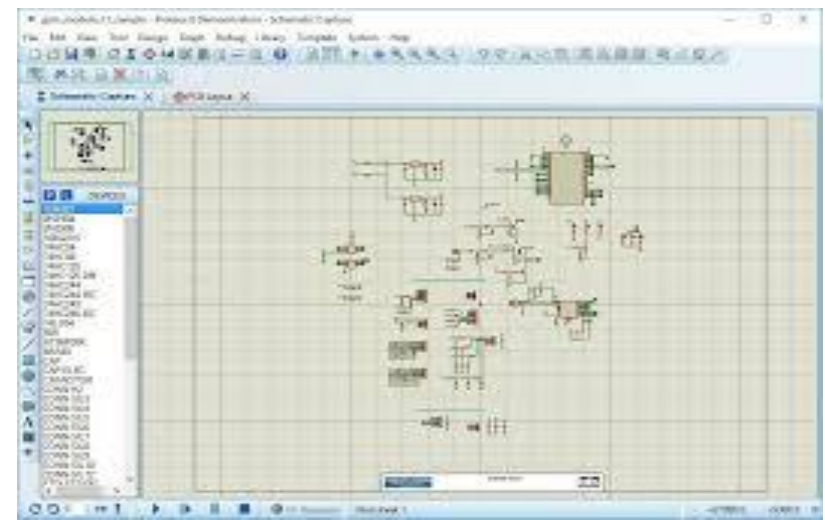

Fig 11: Proteus Design Suite

The Proteus Design Suite is a Windows application for schematic catch, recreation, and PCB (Printed Circuit Board) format plan. It very well may be bought in numerous setups, contingent upon the size of structures being created and the prerequisites for microcontroller reenactment. All PCB Design items incorporate an autorouter and fundamental blended mode SPICE reproduction abilities.
The micro regulator reenactment in Proteus works by applying either a hex document or a troubleshoot record to the microcontroller part on the schematic. It is then corecreated alongside any simple and computerized hardware associated with it. This empowers its utilization in an expansive range of undertaking prototyping in territories, for example, engine control, temperature control and UI plan.

\section{Embedded C}

Installed $\mathrm{C}$ is a lot of language expansions for the $\mathrm{C}$ programming language by the $\mathrm{C}$ Standards Committee to address shared characteristic issues that exist between $\mathrm{C}$ augmentations for various inserted frameworks.

Installed $\mathrm{C}$ programming commonly requires nonstandard augmentations to the $\mathrm{C}$ language so as to help upgraded microchip highlights, for example, fixed-point number juggling, numerous particular memory banks, and essential $\mathrm{I} / \mathrm{O}$ activities. In 2008, the C Standards Committee stretched out the $\mathrm{C}$ language to address such abilities by giving a typical norm to all executions to hold fast to. It incorporates various highlights not accessible in typical $\mathrm{C}$, for example, fixed-point math, named address spaces and essential I/O equipment tending to. Inserted $\mathrm{C}$ utilizes the vast majority of the grammar and semantics of standard $\mathrm{C}$, e.g., primary() work, variable definition, data type revelation, contingent proclamations (if, switch case), circles (while, for), capacities, clusters and strings, structures and association, bit tasks, macros, and so on.

$\mathrm{C}$ is a basic procedural language. It was intended to be ordered utilizing a moderately direct compiler to give low-level admittance to memory and language develops that map proficiently to machine guidelines, all with insignificant runtime uphold. In spite of its low-level abilities, the language was intended to energize cross-stage programming.

\section{Visual Basic}

Microsoft proposed Visual Basic to be moderately simple to learn and utilize. Visual Basic was gotten from BASIC and empowers the quick application improvement (RAD) of graphical UI (GUI) applications, admittance to information bases utilizing Data Access Objects, Remote Data Objects, or ActiveX Data Objects, and making of ActiveX controls and articles.

A developer can make an application utilizing the segments gave by the Visual Basic program itself. After some time the network of software engineers grew outsider parts. Projects written in Visual Basic can likewise utilize the Windows API, which requires outer capacity assertions.

\section{CONCLUSION}

An improvement in submerged correspondence framework is required because of expanded number of automated vehicles in space and submerged. Conventional submerged correspondence depends on acoustic signs and notwithstanding the generous progression in this field, acoustic correspondence is unable to give adequate data 
transmission low inertness. Optical submerged correspondence gives incredible potential to enlarge customary acoustic correspondence because of its high information rates, low dormancy, less force utilization and littler bundling. Likewise, this innovation can profit definitively from the advancement made in the earthly optical remote correspondence. We propose another strategy by adding heartbeats to the FDM technique which is predominantly utilized in submerged wire-less information correspondence. Rather than the regular optical remote transmission, we use information correspondence module.

With this idea we discover a steady answer for observing the wellbeing states of ocean researcher. Now a days, the air conditions are evolving hugely. It influences the ocean pilots in a difficult situation. It is hard to screen their wellbeing conditions when they are in ocean. As we probably am aware, regardless of whether they jump under 20 meters into the ocean their heart beat changes wildly and pulse lows perilously it might prompts passing. Also, this will be hard to guides/angler to continue in those conditions. So here we propose a framework to screen heath states of an ocean guide while checking the heart beat perusing during route. Utilizing an information correspondence framework we can screen pilots heart beat even in ground. So this will assist us with discovering the status of the pilot and can dodge the unsafe circumstances. We can likewise share ready messages to them if the atmosphere changes definitely when they are exploring. This will likewise assist them with understanding the condition and can securely return to the board.

\section{VI.REFERENCES}

[1] C. Pontbriand, N. Farr, J. Ware, J. Preisig, and H. Popenoe, "Diffuse high-bandwidth optical communications", Oceans 2008. IEEE, 2008.

[2] H.G. Rao, C.E. Devoe, A.S. Fletcher, I.D. Gaschits, F. Hakimi, S.A. Hamilton, et al., "Turbid-harbor demonstration of transceiver technologies for wide dynamic range undersea laser communications", Oceans 2016. IEEE, 2016.

[3] Zhang, L., H. Wang and X. Shao, "Improved m-QAM-OFDM transmission for underwater wireless optical communications". Optics Communications, vol. 423: pp. 180-185, 2018.

[4] D. Stramski, A. Bricaud, and A. Morel, "Modelling the inherent optical properties of the ocean based on the detailed composition of the planktonic community". Appl Opt., vol. 40, pp. 2929-2945, 2001.

[5] Jaruwatanadilok, S., "Underwater Wireless Optical Communication Channel Modelling and Performance Evaluation using Vector RadiativeTransfer Theory". IEEE Journal on Selected Areas in Communications, vol. 26(9): pp. 1620-1627, 2008.

[6] Sahu, S.K. and P. Shanmugam, "A theoretical study on the impact of particle scattering on the channel characteristics of underwater optical communication system", Optics Communications, vol 408(SI): pp. 3-14, 2018.

[7] Stojanovic M and Preisig J. Underwater acoustic com-munication channels: propagation models and statistical characterization. IEEE Common Mag 2009; 47(1): 84-89.

[8] Kilfoyle D and Baggeroer A. The state of the art in underwater acoustic telemetry. IEEE J Ocean Eng 2000;25(1): 4-27.

[9] Kaushal H and Kaddoum G. Underwater optical wire-less communication. IEEE Access 2016; 4: 1518-1547.

[10] Zeng Z, Fu S, Zhang H, et al. A survey of underwater optical wireless communications. IEEE CommunSurvTut 2017; 19(1): 204-238. 